\title{
The Study of Structure Formation Processes in the Confectionery Mass
}

\author{
P.M. Smolikhina, E.I. Muratova, S.I. Dvoretsky* \\ Department of Technologies and Equipment for Food and Chemical Production, \\ Tambov State Technical University, 106, Sovetskaya St., Tambov, Russian Federation \\ * Corresponding author. Tel.: + 7 (4752) 6394 42.E-mail: topt@topt.tstu.ru
}

\begin{abstract}
The article is devoted to the complex study of the rheological properties of raw materials, semi-finished and finished products to support optimal modes of production and to manufacture products with specified structural-mechanical characteristics. It was found that the nature of changes in the plastic strength of semi-finished products depends on the modes of tempering and the influence of plant additives on the process of structure formation. The authors also analyze the effect of phytonutrients and vegetable powders on the strength of adhesive contacts between the layers of candy mass in forming multilayered confectionery products. The simulation results of determining the optimal ratio of recipe ingredients, which provide specified structural-mechanical characteristics of the jelly bodies, are presented.

According to the research results, the effect of dosing and dispersion of phytonutrients and vegetable powders on candy mass viscosity at different temperatures and strain rates allowed to develop recommendations for selecting modes of thermomechanical processing of fondant and jelly masses at the stage of tempering and molding.
\end{abstract}

\section{Keywords}

Adhesion; confectionery mass; plastic strength; rheology; structure formation.

(C) P.M. Smolikhina, E.I. Muratova, S.I. Dvoretsky, 2016

\section{Introduction}

Physico-mechanical characteristics are the most important indicators of candy mass properties, where candy mass is a semi-finished product entering further process steps. These characteristics also determine structural and mechanical properties of the finished product. The study of adhesion properties, plastic strength and duration of structure formation process allows to evaluate the possibility of using various additives, technological modes and methods of molding to give the finished products defined properties, stability during transportation and storage.

In recent years domestic and foreign journals have published a number of studies on the rheological behavior of confectionery mass and structure of finished products [1-3].

Constantly expanding the range of products as well as using non-conventional raw materials in candy recipes actualizes the problem of studying the rheological behavior of candy mass and bank data on the influence of recipe ingredients and process conditions on technological properties of semi-finished products and stability of structural and mechanical properties of finished products during the storage period.

The aim of the research is to validate the modes of candy production using enrichers in the form of powders from local vegetable raw materials on the basis of studying rheological, structural and mechanical properties of semi-finished products.

\section{Materials and Methods}

The objects of the study were samples of fondant, jelly and whipped mass and candies made on their basis according to classical recipes, as well as with the addition of vegetable powders (pumpkin, carrot) and medicinal-technical raw materials (nettle leaves, lemon balm, raspberry) of various concentration and dispersion. Herbal additives were used in the form of powder, hydrated powders (puree) and water-alcoholic extracts.

The study of rheological properties of semifinished products and structural-mechanical characteristics of candy bodies were performed on 
HAAKE VT6R plus viscotester (Thermo Fisher Scientific, Germany) and Brookfield CT-3 texture analyzer (Brookfield Engineering laboratories, inc., USA) equipped with a wide range of sensors, devices and accessories to meet the challenges associated with the analysis and measurement of texture. The powder dispersity was measured by a laser particle size analyzer of "Microsizer 201" series. Detailed description of methods for analysis of raw materials, semi-finished and finished products is given in [4].

\section{Results and Discussion}

Studying rheological properties of investigated candy mass has shown that, irrespective of the recipe, they belong to pseudoplastic materials. In a wide range of shear rates $0-100 \mathrm{~s}^{-1}$ the flow of the fondant mass is well approximated by the equation of HerschelBulkley, while the jelly and whipped mass satisfies the equation of Ostwald de Waele. In all cases, the rheological curves are well approximated by a linear function over a fairly wide range of shear rates (by a factor of no less than 0.98 approximation), which explains the choice and use of these rheological equations [4].

Plastic strength of candy bodies is a key indicator for characterizing the form retention quality. Changes in strength can show the process of structure formation, the rate of which is of great importance for selecting regimes for structure formation process of candy mass.

The analysis of experimental studies about the effect of functional ingredients on the process of jelly mass formation showed that the use of powder reduces the plastic strength of jelly by $22 \%$ (Fig. 1).

Introduction of vegetable powders at the tempering stage of the jelly mass leads to destruction of the jelly monolithic structure and formation of an uneven one. Polysaccharides of powders that have high sorption properties violate hydrostatic equilibrium while absorbing moisture from the system, the result of which becomes an irregular jelly body.

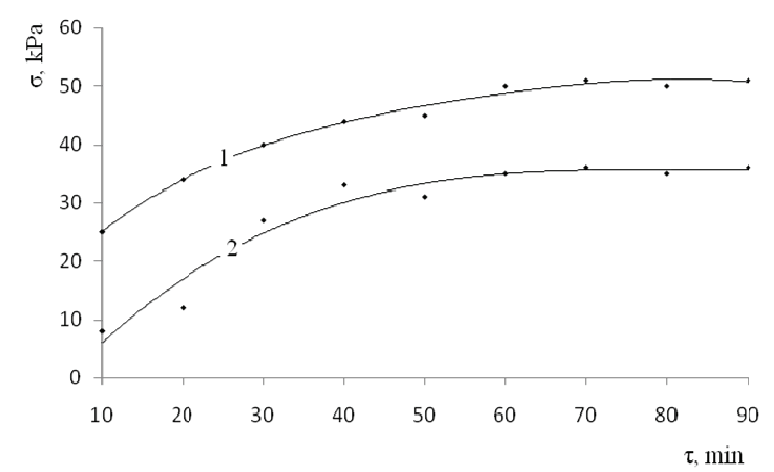

Fig. 1. Changes in the jelly plastic strength during structure formation process:

1 - control; 2 - with the addition of $0.5 \%$ pumpkin powder
When introducing the functional additives with pectin at the syrup preparation stage we can see the maximum dissolving and swelling of the polysaccharide powder. The formed jelly surface is smooth, on the fracture it is glassy with even distribution of insoluble fiber parts (Fig. 2).

To find out the optimal ratio of recipe ingredients in order to obtain a jelly with specified structuralmechanical characteristics and to establish appropriate viscosity value of the jelly mass in the molding process, due to the complexity of the analytical description of the system study on the whole, the authors used methods of mathematical planning of the experiment and the regression analysis for data processing.

As a result, the nonlinear regression equations (1), (2), which adequately describe the dependence of the mass viscosity $y_{1}$ and the jelly plastic strength $y_{2}$ from the concentrations of pectin $\left(x_{1}\right)$, citric acid $\left(x_{2}\right)$ and the concentrated water extract of nettle leaves $\left(x_{3}\right)$, there were obtained:

$$
\begin{gathered}
y_{1}=-14-1,55 x_{1}+5,44 x_{2}+0,68 x_{3}+0,41 x_{1} x_{2}- \\
-0,17 x_{1} x_{3}+0,4 x_{1}^{2}-0,68 x_{2}{ }^{2}-0,02 x_{3}{ }^{2} \\
y_{2}=-233,19+3,79 x_{1}+46,92 x_{2}+25,32 x_{3}+4,73 x_{1} x_{2}- \\
-0,77 x_{1} x_{3}-0,084 x_{2} x_{3}-0,55 x_{1}{ }^{2}-6,4 x_{2}{ }^{2}-1,208 x_{3}{ }^{2} .
\end{gathered}
$$

Maximum misalignment of experimental data and those calculated by model (1) was $2.9 \%$, on the model (2) $-6.7 \%$.

The analysis of equations $(1,2)$ shows that the strength of the effect of these factors on the viscosity of the jelly mass decreases in the series $x_{2}>x_{1}>x_{3}$, and on the plastic strength of jellies $-x_{2}>x_{3}>x_{1}$.

Thus, the greatest impact on the rheological properties of semi-finished jellies is made by citric acid concentration. The effect of pectin and extract concentration is less obvious which demonstrates the importance of jelly mass $\mathrm{pH}$ control at all stages of the production cycle and of its maintaining at the optimal level.

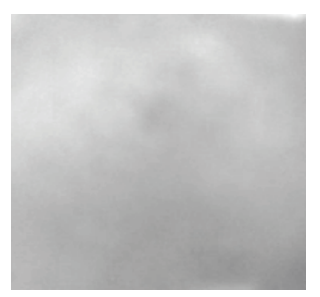

a)

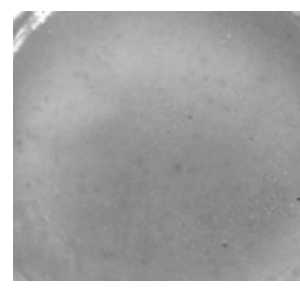

b)

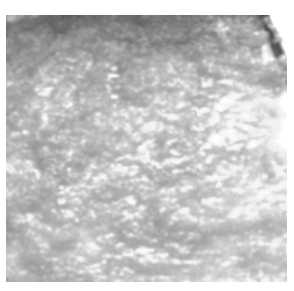

c)
Fig. 2. Jelly mass with the addition of vegetable powders $3 \%$ : $a$-control; $b$ - at the stage of syrup production; $c-$ at the tempering stage 
The graphical representation of the equations (1) and (2) as response surfaces and lines of equal level with a fixed amount of citric acid $x_{2}=4 \mathrm{~g}$ is shown in Fig. 3.

The strength of whipped jellies with the use of powdered semi-finished products, on the contrary, increases due to the combined action of agar molecules and pectin substances presented in vegetable powders in large quantities. Thus, the hydrated powder increases the strength 2.0-fold, the dry powder 8.0 -fold. When using hydrated powder, the whipped mass strength increases owing to additional filling of the space frame surrounding the bubbles with swollen fibers of the vegetable powder. Gelation occurs within 40 minutes after casting at a temperature of $20-22{ }^{\circ} \mathrm{C}$, but a large amount of swollen polysaccharides makes the mass aqueous and prone to syneresis.

In samples containing dry powder syneresis is avoided by narrowing the channel, increasing the roughness of the walls and forming local "gates" from the particles not adhered to the bubbles. However, the presence of solid particles may have the opposite effect: they may undergo the adsorption of surfactants and the concentration decrease of surfactants in the solution leads to the increase in the surface tension and decrease in the foam dispersion, whereby the syneresis speed can be boosted.

Factors affecting the rate of fondant mass structuring are the ratio of solid and liquid phases, the presence of large crystals, the concentration and dispersion of functional additives, and body temperizing modes. The rate of fondant mass structuring can be judged by the increase in the limit shear stress [3].

For classical fondant mass at low temperatures $\left(70-75^{\circ} \mathrm{C}\right)$ the limiting shear stress raises dramatically in a short period of time which indicates a high rate of sucrose crystallization. A high degree of supersaturation of the solution leads to intensive crystallization of sucrose not only on the surface but also in internal layers of the body. Structuring process in the mass casting with temperature of $95^{\circ} \mathrm{C}$ is slower and the mass cast at temperature of $100^{\circ} \mathrm{C}$ reaches normal consistency (critical shear stress of $30-40 \cdot 10^{3} \mathrm{~N} \cdot \mathrm{m}^{-2}$ ) after 3 hours of structure formation process.

The structure formation process of the fondant mass can be traced according to increase in the strength of the structure of candy body. Fig. 4 shows the dependence of the strength of the fondant sample on the depth of the indenter.

On the surface of the semi-finished product there is a dense crystalline crust formation, the hardness of which increases during the first hour (up to $1600 \mathrm{~g}$ ), and after two hours of temporizing the thickness

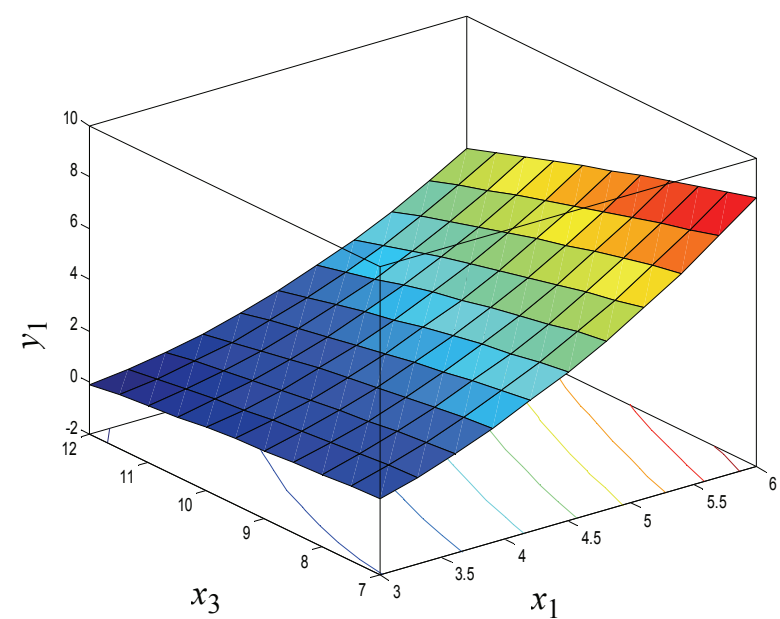

a)

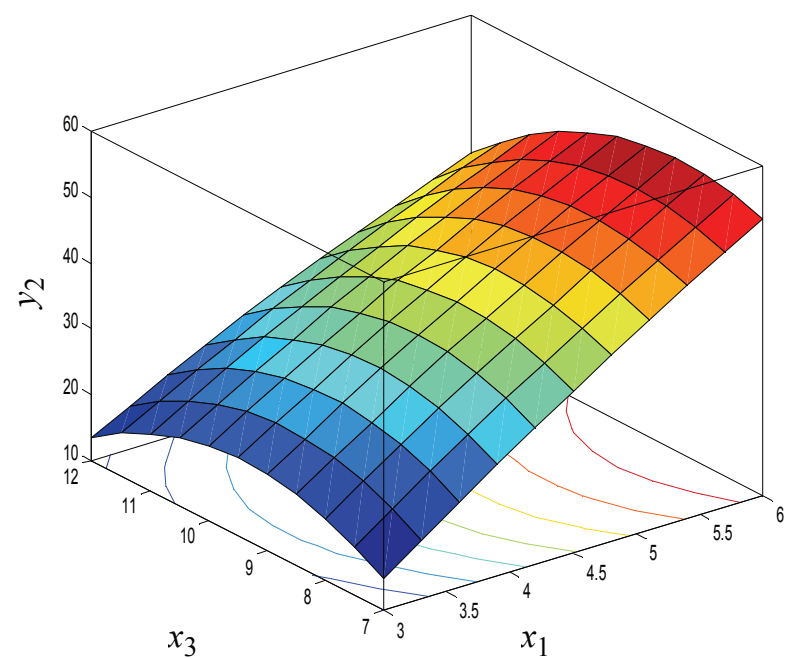

b)

Fig. 3. Surface of goal function response:

$y_{1}$ - jelly mass viscosity, $\mathrm{Pa} \cdot \mathrm{s}(a) ; y_{2}$ - plastic strength of jelly, $\mathrm{kPa}(b)$; depending on factors under study: $x_{1}$ - pectin dosage, $\mathrm{g} ; x_{3}$ - concentrated nettle extract dosage, $\mathrm{g}$

reaches $2.5 \mathrm{~mm}$. Inside the formed body there is thick mass with large crystals of sucrose (the presence of crystals characterizes the presence of peaks within curve) (section $3 *$ ). In the crystallization process the adhesion of samples decreases to stainless steel.

In 2.5-3 hours of structure formation process at ambient temperature of $23-25{ }^{\circ} \mathrm{C}$ the candy body has a solid crystalline structure with the strength $4 \cdot 10^{3} \mathrm{~g}$ (Fig. 5).

The study on adhesive properties of the candy mass helps to evaluate the possibility of using various molding techniques for manufacturing candy bodies.

The violation of production modes, moisture migration between the layers and syneresis during the storage leads to the weakening of the adhesive interactions and changing in structural and mechanical characteristics of the products. 


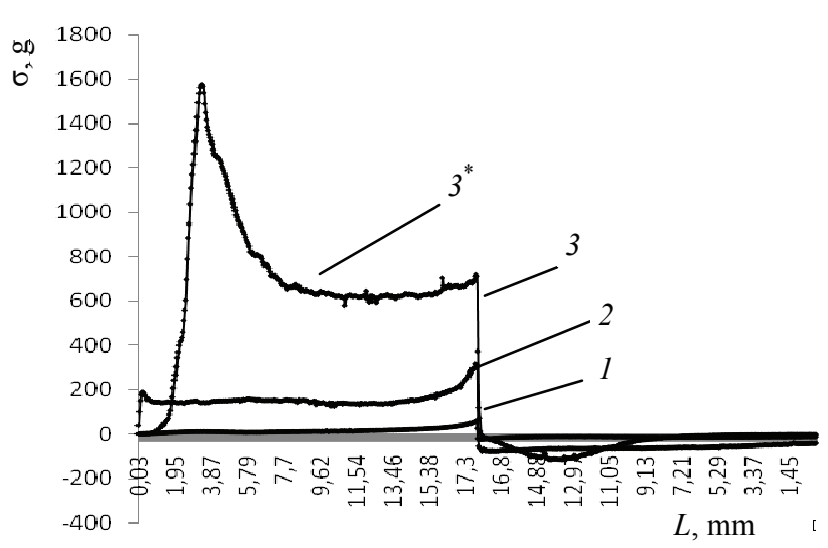

Fig. 4. Changes in the consistency of the fondant mass in the process of structuring after casting in: $1-15 \mathrm{~min} ; 2-35 \mathrm{~min} ; 3-60 \mathrm{~min}$

While forming the adhesive bonding through the combination of jelly and whipped semi-finished products in the combined body, highly viscous masses come into contact. In this case, to describe the process of contact formation rheological characteristics of the adhesive and the terms of the contact become important depending on the method of molding semifinished products.

The molding of combined jelly-whipped candies can be produced with methods of co-extrusion, smearing followed by cutting, and casting.

The practice of molding by co-extrusion method showed that for each production it is necessary to make corrections of technological modes taking into account the constructive features of the molding equipment, or using them for combining confectionery masses with similar rheological characteristics and having thixotropic properties.

The formation of candies by casting and smearing minimizes mechanical effect on the formable candy mass which does not destroy their structure and reduces the residual stress at the phase boundary. The smearing method allows to make multilayer

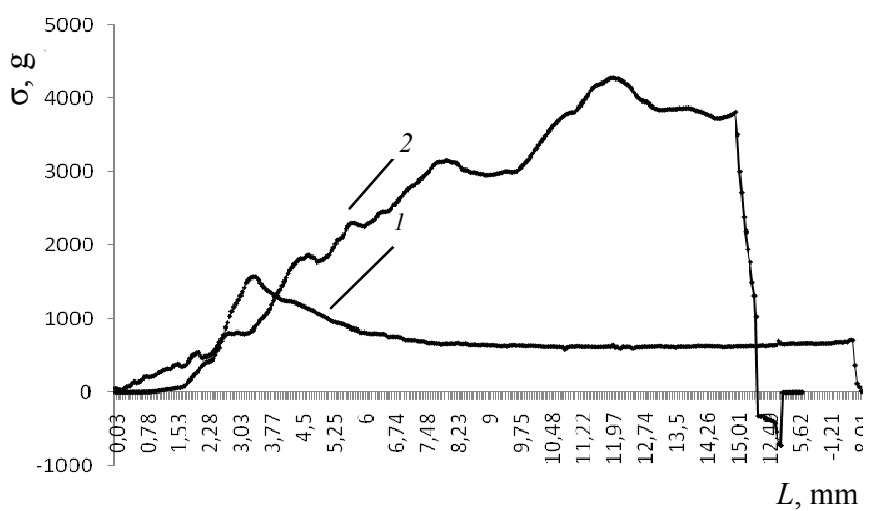

Fig. 5. Changes in the consistency of fondant mass in the process of structuring in: $1-15 \mathrm{~min} ; 2-60 \mathrm{~min}$

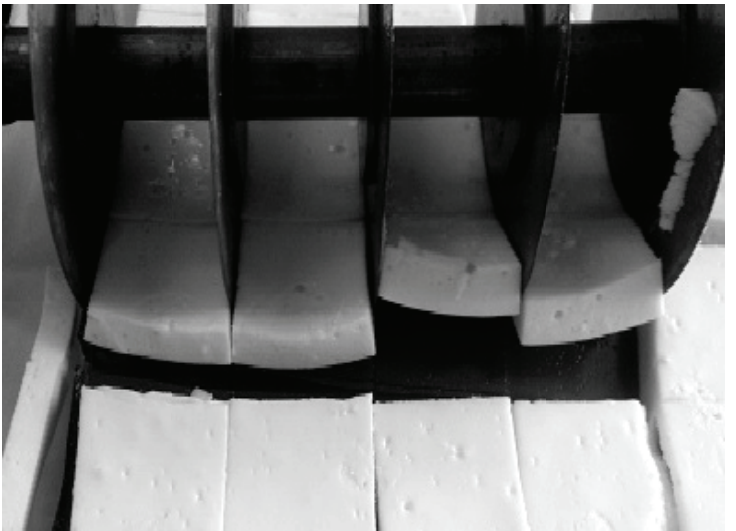

Fig. 6. Adhesion failure in forming combined bodies by smearing and cutting methods

products, but in the process of cutting there may be possible violations of structures, displacement of the layers due to their deformation (Fig. 6).

The formation of jelly-whipped candies was performed by the smearing method followed by cutting and casting. The ability to control rheological properties of the jelly mass by changing the temperature at the molding stage is a determining factor in the choice of jelly layer as the top. When the temperature is increased, the viscosity of the jelly mass decreases non-linearly with average increase in temperature by $1{ }^{\circ} \mathrm{C}$ per $0.01 \mathrm{~Pa} \cdot \mathrm{s}$ [4]. At low viscosity the liquid adhesive wets the surface of the substrate providing a flawless full contact with the whipped mass with the maximum filling of micropores on the surface, but there is a formation of adhesive interaction and hardening of adhesive contact. However, even in this case, the adhesive strength of the contact is insufficient to prevent full separation of the structure by mechanical action on the semi-finished product during cutting the layer obtained by smearing or during removing candy bodies made by the method of casting to the starch forms (Fig. 7).

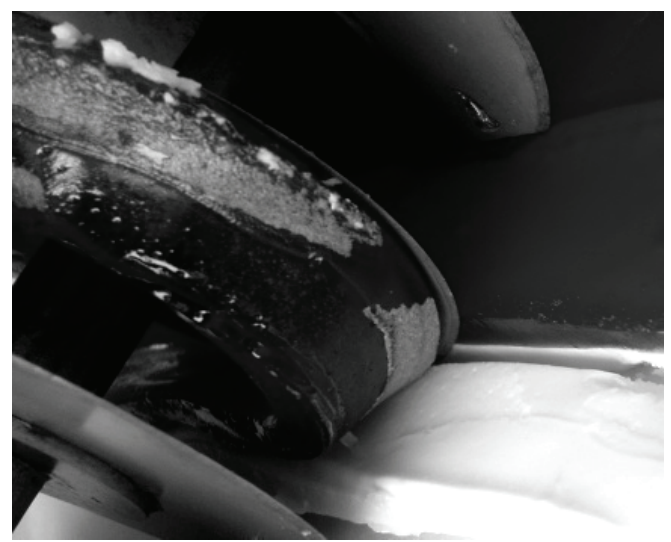

Fig. 7. Mixed destruction of adhesive compound in forming combined candy bodies by smearing and cutting methods 


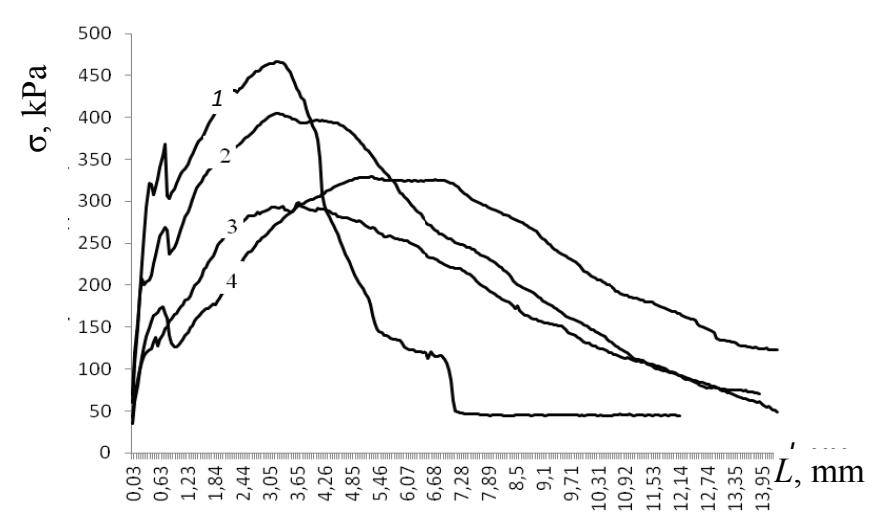

Fig. 8. Dependence of adhesive contacts strength on the depth of separation with the powder in the whipped mass and molding temperature of the jelly layer:

$1-5 \%, 105{ }^{\circ} \mathrm{C} ; 2-5 \%, 95^{\circ} \mathrm{C} ; 3$ - without powders, $95^{\circ} \mathrm{C}$; 4 - without powders, $105^{\circ} \mathrm{C}$

When adding vegetable powders in the whipped layer in the amount of less than $2 \%$, the adhesive strength between the layers of the body does not differ significantly from indicators obtained for the combined bodies without additives. Introducing powders into the whipped layer in the amount more than $10 \mathrm{wt} \%$ leads to excessive development of micro-relief which adversely affects the achievement of maximum contact area: a large number of connections reduces the mobility of macromolecules in the boundary layer, increases internal pressure, changes the structure of the surface layer, which results in defective areas that serve as centers where the destruction of adhesive compounds begins $[3,5]$. The maximum adhesion between the layers of candy mass is attained by adding vegetable powders in the whipped layer in the amount of 5-10 wt \%, in the jelly layer - less than $3 \%$, while the strength of adhesion contacts increases by more than $30 \%$ compared to control samples (Fig. 8, 9).

Based on the above, it can be concluded that adding the carrot powder into the whipped mass allows to improve the contact area of the adhesive-substrate and to increase the adhesive connection strength due to the formation of a rough surface of the whipped mass and maximum filling of microdefects in the jelly mass. Moreover, the persistence of structural and mechanical properties of the whipped mass as a result of enhancing foam frame by rough fibers of the carrot powder provides the structuring capacity of the whipped layer and allows the operation of casting the jelly mass with density of $1350 \mathrm{~kg} / \mathrm{m}^{3}$ at 45-60 minutes after the formation. Vegetable powders having high water-binding capacity absorb moisture from the candy mass surface to improve their adhesion to the combinable layer. Adding powders into the jelly mass reduces jelly strength and allows to change the adhesive tense on its surface which has a positive impact on the quality of the adhesive contact with the whipped mass [5]. The studies have shown that during

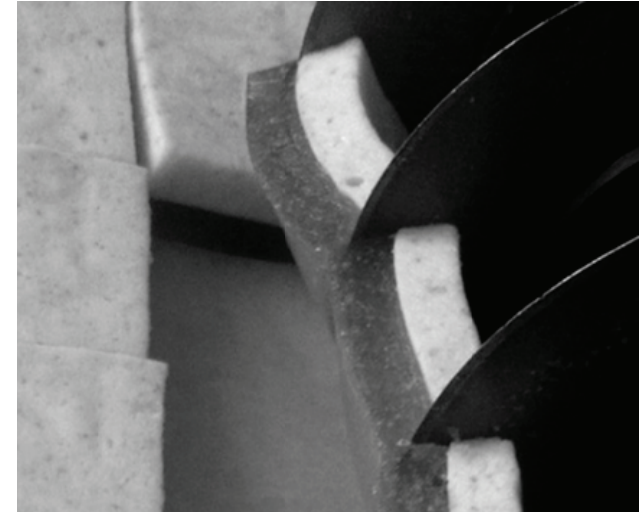

Fig. 9. Cutting of combined candy bodies with $5 \%$ carrot powder in the whipped layer;

$3 \%$ powder pumpkin in the jelly layer

tests of control samples there is usually a mixed adhesive breaking, and that during tests of samples with the addition of vegetable powders the breaking is predominantly cohesive.

\section{Conclusion}

The studies proved operating parameters at different stages of candy production from semifinished products with different rheological properties. It is found that the nature of rheological curves in the studied range of shear rates is similar to candy mass with the addition of powders made from drug-technical and vegetable raw materials with similar values of dispersion indicators, water retention and adsorption capacity.

The design of confectionery products with specified structural-mechanical characteristics as well as the scientific substantiation of the ways of their production is necessary to ensure the technological processes and the optimization of the equipment. This allows to control structural-mechanical characteristics and products quality by introducing additives, changing the modes and methods of mechanical and technological processing.

\section{References}

1. Chetana R., Sripathi K., Sunki Y. Reddy (2004). European Food Research and Technology - EUR FOOD RES TECHNOL, vol. 218 , no. 4 , pp. $345-348$.

2. Kechinski C.P., Carolina P.K., Schumacher A.B., Lígia D., Marczak F., Tessaro I.C., Nilo S., Cardozo M. (2011). Food Hydrocolloids - FOOD HYDROCOLLOID, vol. 25, no. 3, pp. 299-306.

3. Zimon A.D., Yevtushenko A.M. (2008). Adhesion of food masses, M : DeLi print, 398 p. (Rus)

4. Muratowa E.I., Smolikhina P.M. (2013). Rheology of confectionery masses: the monograph, Tambov: Publishing house of FGBOU VPO «TGTU», 188 p. (Rus)

5. Muratowa E.I., Smolikhina P.M. (2013). Proceedings of international scientific and technical Conference after Leonardo da Vinci. Berlin: Wissenschaftliche Welt e. V., no. 1, pp. 177-185. 